\title{
Optimizing Physician Handover Through the Creation of a Comprehensive Minimum Data Set
}

Niraj K. Mistry, Alene Toulany, John F. Edmonds and Anne Matlow

\begin{abstract}
Handover is defined as the communication of information between individuals and teams of healthcare providers to support the transfer of patient care and maintain professional responsibility and accountability. Poor handovers are increasingly recognized as potentially dangerous for patient safety and are associated with adverse events. One suggested method to improve the timely and efficient exchange of clinical information at handover and to reduce discontinuities in care is through the use of a minimum data set (MDS).

The objective of this study was to describe the process of developing a single comprehensive hospital-wide MDS, created through an analysis of current handover processes and customary information tools used to support physician handover (MDHO) at a large quaternary care pediatric academic health sciences centre. A 20-item questionnaire was administered in person to a senior resident or fellow on each of 49 services identified to objectively assess MDHO processes, including frequency, consistency, format, participants and duration, for each service. The presence, type, location, responsibility for updating and security characteristics of MDHO tools used to support MDHO were also analyzed.The MDHO tools currently in use were collected and analyzed to create a comprehensive cross-institutional MDS.

The analysis indicates that MDHO is highly consistent in terms of frequency, processes, participants, duration and the
\end{abstract}

use of written tools to guide information exchange across departments. However, many best practice recommendations for MDHO are not being followed. Further, many of the existing MDHO tools in use have a similar content structure and already contain a majority of the components of a comprehensive MDS.

Current local consistency in practice will allow for improved acceptance and adoption of an MDHO tool that continues to meet the clinical and administrative needs of physicians but also addresses needs for data accuracy and security. These additional specifications can be met through the use of information communication technologies.

\section{Background}

The communication of information to support the transfer of patient care and professional responsibility and accountability, referred to as handover or handoff, is essential to patient safety and occurs commonly in healthcare (Australian Commission on Safety and Quality in Health Care [ACSQHC] 2010). The World Health Organization (WHO 2007) has listed "communication during patient care handover" as one of its "High 5" patient safety initiatives. Aligned with these strategies, Accreditation Canada has identified (handover) communication as one of six patient safety goals in the essential practices to enhance patient safety and minimize risk, known as the required organizational practices (Accreditation Canada 2008). 
Improving effective communication from the time of admission to discharge is also a leading patient safety goal espoused by the Joint Commission (2006) in the United States.

Each individual handover is a potential safety risk to the patient, and, indeed, breakdown in communication is recognized as the leading root cause of sentinel events (WHO 2007). New resident work-hour restrictions are making handovers increasingly frequent among care teams (Kemp et al. 2008; Professional Association of Interns and Residents of Ontario 2008). Ineffective handover can lead to inappropriate treatment, delays in diagnosis, increased healthcare costs and patient morbidity and mortality (Bulau 1992; Petersen et al. 1994; Priest and Holmberg 2000; Pronovost et al. 2002). Growing awareness of the frequency and impact of communication errors in handovers has led to calls for improving their safety and efficacy.

\section{Growing awareness of the frequency} and impact of communication errors in handovers has led to calls for improving their safety and efficacy.

Clinical handover has been a key initiative for the Australian Commission on Safety and Quality, which has completed an extensive, structured and evidenced-based literature review regarding the effectiveness of improvement interventions in clinical handover (Wong et al. 2008). Standardization of clinical handover through the creation of a minimum data set (MDS) was a frequent strategy used in both the quantitative and qualitative studies examined. An MDS refers to the minimum content that must be contained and transferred for an individual patient handover (ACSQHC 2010). There are many possible MDSs for handover; but regardless of the MDS used, this standardization strategy is strongly supported by a recent systematic review of residents' and attending physicians' handovers in the United States (Riesenberg et al. 2009). While a number of MDSs have been developed and implemented, there is little evidence that any of these have been developed through an analysis of information tools already being used for handover (Agency for Healthcare Research and Quality [AHRQ] n.d.; Mikos 2007; Wong et al. 2008).

The objective of this study was to describe the process for the handover of patient information and the content of handover documents used at a quaternary care academic health sciences centre and to identify an MDS that is generalizable across all divisions throughout the organization. This study represents the first phase in our development of a single comprehensive hospital-wide electronic handover tool that is to be embedded within the existing electronic medical record (EMR).

\section{Methods}

\section{Setting}

This study was conducted at The Hospital for Sick Children (SickKids), a 300-bed quaternary care academic health sciences centre in Toronto, Ontario, with pediatric and level III neonatal intensive care units, hematology-oncology and bone marrow and solid/multi-organ transplantation programs.

The hospital Morbidity and Mortality Committee's monthly reviews of safety reports identified physician handover (MDHO) as a potential hazard in the institution, and the medical director of patient safety was charged with assembling a working group to review current processes and make recommendations to improve MDHO by the end of the calendar year (10 months later). At that time, there were no formal policies, guidelines or procedures about the content and processes of MDHO, although relevant policies such as confidentiality of patient information were in existence. Recognizing that MDHO happens at many levels throughout the patient care experience, a multidisciplinary working group was purposively constituted with (1) representation from key areas where MDHO takes place, (2) staff as well as house staff, (3) representation from informatics and (4) those who had previously expressed an interest in the vulnerabilities in the handover procedure. The membership included the medical director of patient safety, who acted as chair (A.M.); a hospitalist, an intensivist, the physician leader of the critical care response team, an anesthesiologist, an emergency room physician, a general surgeon, senior pediatric residents (N.M. and A.T.) and the medical director of informatics (J.E.). The initiative focused solely on multi-patient handovers between physicians, including all levels of trainees and attending staff, that occur for daytime, overnight or weekend coverage.

A quantitative and qualitative approach was undertaken in which questionnaires were used to objectively determine the MDHO process, existing tools were collected and a content analysis was performed to create an MDS. This study was approved at SickKids as a quality improvement project.

\section{Participants}

Of the 86 medical surgical and diagnostic services identified, 37 operated on daytime schedules only (e.g., pathology, laboratory medicine etc.) and did not participate in MDHO, leaving 49 services appropriate for questioning. A senior resident or fellow who had direct involvement in the MDHO process on that service during a one-week period in September 2009 was identified and approached to be surveyed.

\section{Questionnaire}

A 15-item MDHO processes questionnaire was constructed that included a combination of multiple-choice and open-ended questions to objectively assess MDHO frequency, consistency, 
process, participants and duration. An additional five multiple-choice questions were used to determine the presence, type, location, responsibility for updating and security characteristics of the tools used to support MDHO (Appendix 1, http:// www.longwoods.com/content/ 21925). The questionnaires were pilot-tested for content validity, structure and clarity among the co-authors and with two colleagues. The survey was administered to each designated physician face to face by one of the authors and participation was voluntary and anonymous as identifying information was not collected.

Representatives from each of the 49 services eligible for participation completed the survey, representing a response rate of $100 \%$.

\section{MDS Generation}

Following the questionnaire administration, a hard copy of the current, most up-to-date version of the MDHO tool used by each service was collected for analysis. Of the 49 services identified, 30 services maintained a regular patient list, which was analyzed for formatting (horizontal versus vertical orientation, number of columns, number of pages, number of patients, presence of a header or footer and description of section titles).

\section{Results}

\section{MDHO Processes Questionnaire}

Of the 49 services identified, seven services were surgical and 42 were medical. Thirtyfive services consistently conducted MDHO twice daily, four services handed over once daily and 10 services handed over on an as-required basis depending on the patient census and number of physicians on service (Table 1). Overall, MDHO was quite consistent within each service. Most morning MDHOs took place between 6:15 and 8:30 on weekdays and between 6:30 and 9:00 on weekends. Evening MDHOs took place between 16:30 and 17:30 on weekdays and between 14:00 and 17:30 on weekends. $\mathrm{MDHO}$ was also very consistent in terms of where it took place
Table 1. MDHO processes questionnaire results for 35 services that handover twice daily

\begin{tabular}{|c|c|c|}
\hline \multirow[b]{2}{*}{ Question } & \multicolumn{2}{|c|}{$\begin{array}{l}\text { Number of Services } \\
\text { Answering "All/Most of } \\
\text { the Time" (\%) }\end{array}$} \\
\hline & a.m. & p.m. \\
\hline In general, how often does MDHO take place? & $32(91)$ & $34(97)$ \\
\hline How consistently does MDHO take place at these times? & $35(100)$ & $35(100)$ \\
\hline \multicolumn{3}{|l|}{ Where and how does MDHO usually take place? } \\
\hline Face-to-face (verbal): & $30(86)$ & 31 (89) \\
\hline On a ward & $13(43)$ & $15(48)$ \\
\hline In a conference room & $8(27)$ & $4(13)$ \\
\hline During walk-around rounds & $3(10)$ & $2(7)$ \\
\hline In the trainee lounge or office & $6(20)$ & $10(32)$ \\
\hline Over the phone (verbal) & $4(11)$ & $3(9)$ \\
\hline Electronically via e-mail or intranet list (non-verbal) & $1(3)$ & $1(3)$ \\
\hline How consistently does MDHO take place in this way? & $30(86)$ & $34(97)$ \\
\hline \multicolumn{3}{|l|}{ Who attends MDHO most often? } \\
\hline Residents only & $7(20)$ & $4(11)$ \\
\hline Residents and fellows & $7(20)$ & $8(23)$ \\
\hline Fellows only & $12(34)$ & $15(43)$ \\
\hline Residents, fellows and nurse practitioners & $3(9)$ & $4(11)$ \\
\hline Residents, fellows and staff & $5(14)$ & $2(6)$ \\
\hline Residents, fellows, nurse practitioners and staff & $1(3)$ & $2(6)$ \\
\hline \multicolumn{3}{|l|}{ How long does MDHO usually take? } \\
\hline$<5$ minutes & $4(11)$ & $4(11)$ \\
\hline $5-15$ minutes & $16(46)$ & $9(26)$ \\
\hline $16-30$ minutes & $11(31)$ & $13(37)$ \\
\hline $31-45$ minutes & $0(0)$ & $6(17)$ \\
\hline$>45$ minutes & $4(11)$ & $3(9)$ \\
\hline
\end{tabular}

$\mathrm{MDHO}=$ physician handover. 
Table 2. MDHO tools questionnaire results for $\mathbf{3 0}$ electronically generated service lists*

\begin{tabular}{|l|c|}
\hline Characteristic & Number of Lists (\%) \\
\hline Location of list & $13(43)$ \\
\hline Public drive (list accessible from any computer on intranet) & $7(23)$ \\
\hline Private drive (list accessible from enabled computers on intranet) & $5(17)$ \\
\hline Computer (accessible on certain computers only) & $5(17)$ \\
\hline Data warehouse & \\
\hline Type of list & $24(80)$ \\
\hline Word processing program (Microsoft Word) & $1(3)$ \\
\hline Computerized spreadsheet program (Microsoft Excel) & $5(17)$ \\
\hline Database program (data warehouse) & $20(67)$ \\
\hline Responsible for updating list & $10(33)$ \\
\hline Residents only & $3(10)$ \\
\hline Residents and fellows & $5(17)$ \\
\hline Residents, fellows and nurse practitioners & $9(30)$ \\
\hline Residents and medical students & $5(17)$ \\
\hline Fellows only & \\
\hline Fellows and nurse practitioners & \\
\hline Security of list (password protected) & \\
\hline Yes & \\
\hline No & \\
\hline
\end{tabular}

$\mathrm{EMR}=$ electronic medical record; $\mathrm{MDHO}=$ physician handover.

${ }^{*} \mathrm{~A}$ total of 39 (of 49 [80\%]) lists were electronically generated. Nine of these were linked to EMRs. The remaining 30 (61\%) outlined in this table were not EMR linked. which was not available for review. Thus, 39 electronically maintained and paper-printed lists to support MDHO were available for analysis. All lists were updated for each handover by residents or fellows and rarely by attending physicians (Table 2).

Of the 39 lists, nine used common vendor-supplied filters built into the existing EMRs to generate patient lists that contained minimal demographic and administrative information (location, name, date of birth and medical record number), a single admitting diagnosis and the name of the primary responsible physician. These nine lists were secure as they were generated from within the password-protected environment of the EMRs and did not require user input, aside from the maintenance of a current list of in-patients on each service.

The remaining 30 lists were electronically generated and not EMR linked (Table 2 ). They were individually maintained by the medical students, residents, nurse practitioners and fellows on each service, and accessible from all computers on the hospital intranet or designated computers with access to a specific shared virtual hard drive (see Table 2). Twenty MDHO lists were password protected. Microsoft SharePoint - a content management system that allows the setup of a centralized, password-protected space for sharing Microsoft Office documents - was used by two services, and their lists were accessible only from designated computers within their department's trainee workrooms or on the ward. Finally, one division had recently designed and implemented an

Most morning MDHOs lasted 15 minutes or less, whereas most evening MDHOs took longer. MDHOs took place in various locations, including the ward nursing stations and the trainee office or lounge. MDHOs were done by telephone, e-mail or using computerized records.

\section{MDHO Tools Questionnaire}

Of the 49 services surveyed, eight did not maintain a regular patient list; one list contained information for two services within the same division. Another service used an entirely handwritten list maintained by the person on call each day,
MDHO list generated from its patient database system that imported demographic information, current problem lists and treatment protocols, with an additional area for free-text entry.

Interestingly, a majority of services within SickKids are using various electronic MDHO tools to support verbal information exchange with visual data at MDHO. While these tools may meet the clinical and administrative needs of physicians, they were not without issues. The majority of MDHO systems in use were not secure. As many as one third of the MDHO lists were not password protected; and among those lists that were, the passwords were not unique to each user and, in some cases, 
a single password was used across multiple service lists. Additionally, a majority of the lists could be accessed on any computer on the hospital intranet. The information completeness and accuracy of the handover lists was not assessed; however, given that all data had to be manually entered into the various electronic MDHO lists, there were likely transcriptional errors.

\section{MDS Generation}

All the lists except one were organized in a horizontal tabular format, with a majority having five to six columns (Table 3); one list was written in paragraph format. On average, each list was close to three pages long and contained information for about 13 patients. Over half of the lists were organized by patient location, including floor and room number, while the remaining lists were organized in alphabetical order according to patient name. Nearly all the MDHO lists had a header or footer that contained administrative details and information about the care providers, including the service name, members of current service team and contact information together with other service-specific useful phone numbers. Finally, over three quarters of the lists contained four of the major sections (Table 3).

Using this information and a modified Delphi method, the content of what the working group considers a generalizable, hospital-wide standardized handover MDS was created (Table 4). Aside from those broad categories and subcategories contained in Table 3, additional subcategories include review frequency, resuscitation concerns and the date and time of last update. Members of the working group agree that it is important to identify those patients who are a priority for review and who have the potential to deteriorate. Furthermore, if there are patients who have any risk factors for a difficult resuscitation, it is important for the oncoming physicians to be aware of those issues. Finally, the need to know the precise time that the MDHO list was updated is also
Table 3. Minimum dataset generation from MDHO content analysis for 30 electronically generated service lists*

\begin{tabular}{|l|c|}
\hline Characteristic & $\begin{array}{c}\text { Number of } \\
\text { Lists (\%) }\end{array}$ \\
\hline Horizontal orientation & $25(83)$ \\
\hline Average number of columns (mean \pm SD) & $5.7 \pm 2.0$ \\
\hline Average number of patients (mean \pm SD) & $12.7 \pm 8.9$ \\
\hline Average number of pages (mean \pm SD) & $2.9 \pm 3.0$ \\
\hline Presence of header or footer & $28(93)$ \\
\hline Section titles & \\
\hline Demographic and administrative information & 30 (100) \\
\hline HPI/PMHx/diagnosis/presentation/issues/problems & $27(90)$ \\
\hline Laboratory and other investigations/significant results/pending tests & 9 (30) \\
\hline Medications/treatments/procedures/diet/fluids/tubes/lines/drains & $23(77)$ \\
\hline Plan/follow-up/to-do tasks & $26(87)$ \\
\hline
\end{tabular}

$\mathrm{HPI}=$ history of presenting illness; $\mathrm{MDHO}=$ physician handover; $\mathrm{PMHX}=$ past medical history; $\mathrm{SD}=$ standard deviation . *Not linked to electronic medical records.

Table 4. Standardized physician handover list minimum dataset for SickKids

\begin{tabular}{|c|}
\hline Column 1: Patient demographic and administrative information \\
\hline Name \\
\hline Medical record number \\
\hline Location (ward, room number) \\
\hline Date of birth \\
\hline Date of arrival/length of stay \\
\hline Anthropometrics: weight, height \\
\hline Review frequency \\
\hline Resuscitation concerns (e.g., Rapid Response Team following, difficult airway etc.) \\
\hline Date and time of last update \\
\hline Column 2: HPI/PMHx/diagnosis/presentation/issues/problems \\
\hline Column 3: Laboratory and other investigations/significant results/pending tests \\
\hline Column 4: Medications/treatments/procedures/diet/fluids/tubes/lines/drains \\
\hline Column 5: Plan/follow-up/to-do tasks \\
\hline
\end{tabular}

$\mathrm{HPI}=$ history of presenting illness; $\mathrm{PMHx}=$ past medical history 
Table 5. Summary of MDHO Best Practice Strategies and Recommendations

\author{
Standardization \\ Standardized process for MDHO using specific, agreed-upon \\ techniques including mnemonics if suitable \\ Preparation - a formally recognized plan instituted at the end of \\ a shift or change in service with adequate time during the shift \\ dedicated for verbal exchange of information \\ Verbal exchange of patient information that includes: \\ Face-to-face communication: \\ III patients are given priority \\ Insight on what to anticipate or what to do \\ Read-back - ensure an interactive process, two-way \\ communication \\ Flexibility to discuss anticipated events, recommendations and \\ ask questions \\ Content exchange summary that includes the following aspects: \\ Standardized content/template or a technological solution \\ should be used for accessing and recording patient \\ information \\ Inclusion of all patients to be handed over \\ Available in a centralized location \\ All data kept up to date in both completeness and accuracy \\ Anticipated events clearly labelled \\ Action items highlighted
}

\section{Communication skills}

Improve general communication skills to overcome language and ethnic barriers

Limit hierarchy and social barriers

Provide training and education on handover expectations, especially to new users

Evaluate the handover process

\section{Physical environment}

Location - private space to avoid breeching patient confidentiality

Limit interruptions, distractions and noise

Address physical environment - lighting issues, space to write

Recognize transfer of responsibility and accountability

$\mathrm{MDHO}=$ physician handover.

felt to be important to patient safety and communication.

\section{Discussion}

A structured and standardized approach, including an MDS, for physician-to-physician handover is recognized as critical to improving patient safety during care transitions (Arora et al. 2009; Patterson et al. 2004; Riesenberg et al. 2009; Wong et al. 2008). While a number of MDS and standardized protocols exist, they are meant to provide structure to the MDHO process, and their use and implementation by hospitals may require more specific details than those provided (AHRQ n.d.; Mikos 2007; Wong et al. 2008). Thus, we completed this study to better understand the local environment and current practices for MDHO.

Some key principles were invoked in developing a local standardized handover MDS. First, key stakeholder involvement was enlisted by ensuring that representatives from key disciplines, such as trainees as well as attending physicians, participated on the working group. Second, our goal was to build on existing structures and processes; thus, an internal scan of existing practices allowed us to harness similarities and to assess the degree of change that was going to be incurred in $\mathrm{MDHO}$ across the organization.

\section{The benefits of an EMR-integrated $\mathrm{MDHO}$ tool are numerous.}

As a result of this process, we recognize that MDHOs at SickKids are very consistent in terms of frequency, consistency, process, participants, duration and use of written tools to guide information exchange. However, many best practices recommendations are not being followed (Table 5) (ACSQHC 2010; Arora et al. 2009; Riesenberg et al. 2009; Wong et al. 2008). MDHO takes place in physical settings where distractions occur and patient privacy and confidentiality might be violated. Direct face-to-face communication, which is almost always preferred (Solet et al. 2005), is lacking in some MDHOs, denying the participants the appreciation of facial expressions and body language, which provide additional information about the level of concern regarding a patient's needs.

Perhaps our most significant finding was that many of the existing MDHO tools already contain components of a comprehensive MDS (Tables 3 and 4). This local consistency in practice will allow for improved acceptance and adoption of an MDHO tool that continues to meet the clinical and administrative needs of physicians, but also affords increased data accuracy through decreased transcriptional errors and increased security and confidentiality. These additional specifications can be met through the use of technology.

The use of information communication technologies, such as electronic handover tools, has been suggested to help reduce communication errors and adverse events and to improve the timely and convenient exchange of clinical information during handover (Petersen et al. 1998). As such, our next steps are to use information technology system design methodologies to create an $\mathrm{MDHO}$ tool embedded within our existing commercial EMR system. The benefits of an EMR-integrated MDHO tool include improving information completeness and legibility, 
compliance with privacy legislation and data security, improved accuracy by mitigating the need for repeated manual data transcription, remote accessibility and the fact that these tools are preferred by residents when compared with written handovers (Anderson et al. 2010; Bernstein et al. 2010; Peterson et al. 1998; Ram and Block 1992; Volpp and Grande 2003). There is also the potential added benefit of accessibility by all members of the healthcare team, improving inter-professional communication between physicians, nurses and other allied healthcare professionals (Sidlow and Katz-Sidlow 2006). Additional functionalities will include the ability to print an MDHO list so it is readily accessible; to sort the list by ward, bed number or physician; and to highlight high-priority patients to be reviewed and tasks needing urgent completion (Cheah et al. 2005).

\section{While a number of MDS and}

\section{standardized protocols exist, their use and implementation by hospitals may require more specific details than those provided.}

\section{Limitations}

This study has several limitations. The scope was restricted to intra-departmental MDHOs and did not include handovers across different departments, disciplines or institutions, perhaps limiting the generalizability of the results to other clinical care transition interfaces. Furthermore, the survey methodology used facilitated the inclusion of many care interfaces, while denying access to the rich qualitative data that may have further informed development of the tool. Also, participants may have altered their answers because the interviewer was present during the questionnaire completion.

\section{Conclusion}

Today's healthcare environment is very complex and interconnected and, as a result, not conducive to prescriptive interventions. As such, the need for flexible standardization through adaptive systems that take into account the local processes and culture is an integral component of ensuring effective, efficient and safe healthcare. In the future, perhaps strategies involving both providers and patients/families in the handover process may prove to be the ultimate way to improve communication during MDHO. Patients and their families are the only constant within this system and may therefore be in position to play critical roles in ensuring the safest and best-quality healthcare (WHO 2006). HQ

\section{References}

Accreditation Canada. 2008. Required Organizational Practices. Ottawa, ON: Author. Retrieved April 7, 2010. <http://www.accreditation-canada.ca/default .aspx? page $=355 \&$ cat $=30>$.
Agency for Healthcare Research and Quality, US Department of Health and Quality. n.d. TeamSTEPPS: National Implementation. Rockville, MD: Author. Retrieved June 19, 2010. <http://teamstepps.ahrq.gov>.

Anderson, J., D. Shroff, A. Curtis, N. Eldridge, K. Cannon, R. Karnani et al. 2010. "The Veterans Affairs Shift Change Physician-to-Physician Handoff Project." Joint Commission Journal on Quality Improvement 36(2): 62-71

Arora, V.M., E. Manjarrez, D.D. Dressier, P. Basaviah, L. Halasyamani and S. Kripalani. 2009. "Hospitalist Handoffs: A Systematic Review and Task Force Recommendations." Journal of Hospital Medicine 4(7): 433-40.

Australian Commission on Safety and Quality in Health Care. 2010. The OSSIE Guide to Clinical Handover Improvement. Sydney, Australia: Author.

Bernstein, J.A., D.L. Imler, P. Sharek and C.A. Longhurst. 2010. "Improved Physician Work Flow after Integrating Sign-Out Notes into the Electronic Medical Record.” Joint Commission Journal on Quality Improvement 36(2): 72-78.

Bulau, J.M. 1992. "Alleged Failure to Treat a Technology-Dependent Infant in the Home Health Care Setting: A Risk Management Case Study." Journal of Home Health Care Practice 5(1): 47-54.

Cheah, L. D.H. Amott, J. Pollard and D.A.K. Watters. 2005. "Electronic Medical Handover: towards Safer Medical Care." Medical Journal of Australia 183(7): 369-72.

Joint Commission. 2006. Critical Access Hospital and Hospital National Patient Safety Goals. Oakbrook Terrace, IL: Author. Retrieved June 19, 2010. <http://www.jointcommission.org/PatientSafety/ NationalPatientSafetyGoals/06_npsg_cah.htm>.

Kemp, C.D., J.M. Bath, J. Berger, A. Bergsman, T. Ellison, K. Emery et al. 2008. "The Top 10 List for a Safe and Effective Sign-Out." Archives of Surgery 143(10): 1008-10.

Mikos, K. 2007. "Monitoring Handoffs for Standardization.” Nursing Management 38: 16-20.

Patterson, E.S., E.M. Roth, D.D. Wood, R. Chow and J. Orlando Gomes. 2004. "Handoff Strategies in Setting with High Consequences for Failure: Lessons for Health Care Operations." International Journal for Quality in Health Care 16(2): 125-32.

Peterson, L.A., E.J. Orav, J.M. Teich, A.C. O’Neil and T.A. Brennan. 1998. "Using a Computerized Sign-Out Program to Improve Continuity of Inpatient Care and Prevent Adverse Events." Joint Commission Journal on Quality Improvement 24(2): 77-87.

Petersen, L.A., T.A. Brennan, A.C. O’Neil, E.F. Cook and T.H. Lee. 1994. "Does Housestaff Discontinuity of Care Increase the Risk for Preventable Adverse Events?" Annals of Internal Medicine 121(11): 866-72.

Priest, C.S. and S.K. Holmberg. 2000. "A New Model for the Mental Health Nursing Change of Shift Report." Journal of Psychosocial Nursing and Mental Health Services 38(8): 36-43.

Professional Association of Interns and Residents of Ontario. 2008. "Article 16: Maximum Duty Hours." In 2008-2011 PAIRO-CAHO Agreement. Retrieved April 11, 2010. <http://www.pairo.org/Content/ Files/2008\%20PAIRO-CAHO.pdf>.

Pronovost, P., A.W. Wu, T. Dorman and L Morlock. 2002. "Building Safety into ICU Care." Journal of Critical Care 17(2): 78-85.

Ram, R. and B. Block. 1992. "Signing Out Patients for Off-Hours Coverage: Comparison of Manual and Computer-Aided Methods." Proceedings of the Annual Symposium of Computer Applications in Medical Care 114-18. 
Riesenberg, L.A., J. Leitzsch, J.L. Massucci, J. Jaeger, J.C. Rosenfield, C. Patow et al. 2009. "Residents' and Attending Physicians' Handoffs: A Systematic Review of the Literature." Academic Medicine 84(12): 1775-83.

Sidlow, R. and R.J. Katz-Sidlow. 2006. "Using a Computerized Sign-Out System to Improve Physician-Nurse Communication." Joint Commission Journal on Quality Patient Safety 32: 32-36.

Solet, D., J.M. Norvell, G. Rutan and R. Frankel. 2005. "Lost in Translation: Challenges and Opportunities in Physician-to-Physician Communication during Patient Handoffs." Academic Medicine 80(12): 1094-99.

Volpp, K.G.M. and D. Grande. 2003. "Residents' Suggestions for Reducing Errors in Teaching Hospitals." New England Journal of Medicine 348: 851-55.

Wong, M.C., K.C. Yee and P. Turner. 2008. Clinical Handover Literature Review. Tasmania, Australia: eHealth Services Research Group, University of Tasmania.

World Health Organization Collaborating Centre for Patient Safety Solutions. 2007. Communication during Patient Handovers. Geneva, Switzerland: WHO Press.

World Health Organization Collaborating Centre International Steering Committee. 2006 London Declaration, Patients for Patient Safety, WHO World Alliance for Patient Safety. Geneva, Switzerland: Author. Retrieved April 7, 2010. <http://www.who.int/patientsafety/ information_centre/London_Declaration_May06.pdf>.

\section{About the Authors}

Niraj K. Mistry, MD, is a member of the Department of Paediatrics at The Hospital for Sick Children and the University of Toronto, Toronto, Ontario.

Alene Toulany, MD, is a member of the Department of Paediatrics at The Hospital for Sick Children and the University ofToronto.

John F. Edmonds, MD, FRCPC, is a member of medical informatics, technology and information services at The Hospital for Sick Children and the University of Toronto.

Anne Matlow, MD, FRCPC, is a member of the Department of Paediatrics at The Hospital for Sick Children and the University ofToronto; and the Centre for Patient Safety at the University of Toronto. You can contact Dr. Matlow by phone at 416-813-5966, by fax at 416-813-4992 or by e-mail at anne. matlow@sickkids.ca.

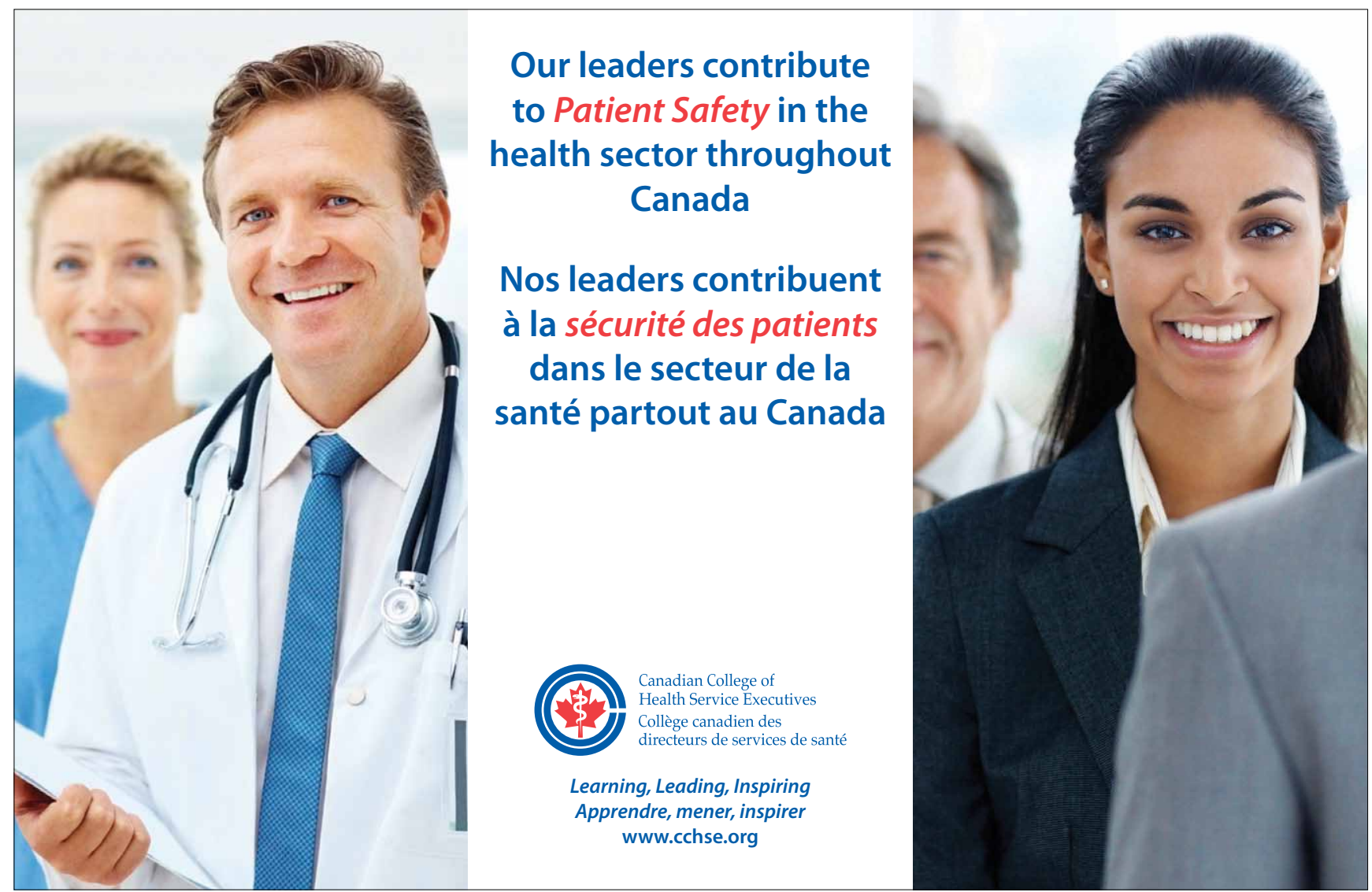




\section{Shoe Leather Handover Survey: Phase I}

Date: September , 2009

Clinical handover is a key element of Patient Safety and we were hoping to take 10 minutes to ask you a few questions about the department/services' current handover processes and handover tools. We would also like to collect the most recent, updated copy of your handover list, if one exists. Please note that your particip ation is completely voluntary and your participation will have no effect on your work evaluation. Your responses will remain anonymous and only the interviewer will know your identity. This has been approved as a Quality Improvement project.

Service/Department Name:

Service Type: $\quad$ Medical Surgical $\quad$ Other

Level of Training of Interviewee:

\section{$\underline{\text { Physician Handover Processes }}$}

1) On average, how many times in 24 hours does handover (physician-to-physician, not rounds) occur?

0 times

1 time

2 times

$\geq 3$ times

\section{AM Handover}

2) In general, how often does AM handover take place?

Never Sometimes Most of the time All the time

3) At what times does AM handover usually take place?

on weekdays:

on weekends:

4) How consistently does AM handover take place at these times?

Never Sometimes Most of the time All the time

5) Where and how does AM handover usually take place? (Circle all that apply)

\begin{tabular}{|c|c|c|c|c|c|c|}
\hline $\begin{array}{l}\text { on the ward } \\
\text { (nursing } \\
\text { station) } \\
\text { face-to-face }\end{array}$ & $\begin{array}{l}\text { conference } \\
\text { room } \\
\text { face-to-face }\end{array}$ & $\begin{array}{l}\text { walk-around } \\
\text { rounds } \\
\text { face-to-face }\end{array}$ & $\begin{array}{l}\text { Lounge } \\
\text { face-to-face }\end{array}$ & $\begin{array}{l}\text { Trainee } \\
\text { office } \\
\text { face-to-face }\end{array}$ & $\begin{array}{l}\text { Over the } \\
\text { phone }\end{array}$ & $\begin{array}{l}\text { Electronically } \\
\text { (via email, } \\
\text { MSN, } \\
\text { facebook) }\end{array}$ \\
\hline
\end{tabular}

Other:

6) How consistently does AM handover take place at these locations and in this way? 
Never Sometimes Most of the time All the time

7) Who attends AM handover most often? (Circle all that apply)

Medical Student $\quad$ Resident $\quad$ Fellow $\quad$ Nurse Practitioner $\quad$ Staff physician

Other:

8) How long does AM handover usually take?

$<5$ minutes $\quad 5-15$ minutes $\quad 16-30$ minutes $\quad 31-45$ minutes $\quad>45$ minutes

\section{PM Handover}

9) In general, how consistently does PM handover take place:

Never Sometimes Most of the time All the time

10) At what times does PM handover usually take place?

on weekdays:

on weekends:

11) How consistently does PM handover take place at these times?

Never Sometimes Most of the time All the time

12) Where and how does PM handover usually take place? (Circle all that apply)

\begin{tabular}{|c|c|c|c|c|c|c|}
\hline $\begin{array}{l}\text { on the ward } \\
\text { (nursing } \\
\text { station) } \\
\text { face-to-face }\end{array}$ & $\begin{array}{l}\text { conference } \\
\text { room } \\
\text { face-to-face }\end{array}$ & $\begin{array}{l}\text { walk-around } \\
\text { rounds } \\
\text { face-to-face }\end{array}$ & $\begin{array}{l}\text { Lounge } \\
\text { face-to-face }\end{array}$ & $\begin{array}{l}\text { Trainee } \\
\text { office } \\
\text { face-to-face }\end{array}$ & $\begin{array}{l}\text { Over the } \\
\text { phone }\end{array}$ & $\begin{array}{l}\text { Electronically } \\
\text { (via email, } \\
\text { MSN, } \\
\text { facebook) }\end{array}$ \\
\hline
\end{tabular}

Other:

13) How consistently does PM handover take place at these locations and in this way?

Never Sometimes Most of the time All the time

14) Who attends PM handover most often? (Circle all that apply)

Medical Student $\quad$ Resident $\quad$ Fellow $\quad$ Nurse Practitioner $\quad$ Staff physician

Other: 
15) How long does PM handover usually take?

$<5$ minutes $\quad 5-15$ minutes $\quad 16-30$ minutes $\quad 31-45$ minutes $\quad>45$ minutes

\section{$\underline{\text { Physician Handover Tools }}$}

16) Does your service have a handover list? YES NO N/A

17) Is this list: electronically generated, but printed out (eg. Word, Excel, KidCare, CIMS, Data warehouse)

electronically generated, but NOT printed (ie. Electronic list is passed from person to person)

not electronic, hand written

not electronic, not hand written, verbal handover only

18) Where is the list located?

shared hard-drive designated computer data warehouse list CIMS list kidcare list only

Other:

19) If the list is electronic, is the list password protected? YES NO N/A

20) Who is usually responsible for updating the list? (Circle all that apply)

Medical Student $\quad$ Resident $\quad$ Fellow $\quad$ Nurse Practitioner $\quad$ Staff physician 


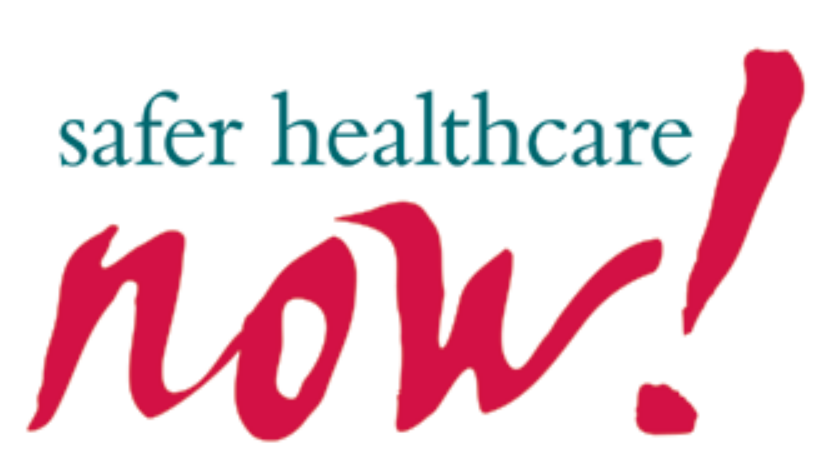

Reducing Harm Improving Healthcare Protecting Canadians

\section{Come learn with Safer Healthcare Now!}

Safer Healthcare Now! is hosting several virtual learning programs in 2010-2011! The goal of these programs is to guide teams in implementing patient safety improvement projects from the convenience of their own workspace!

\section{Starting in Fall 2010}

\section{Virtual Action Series':}

- New Approach to Controlling Superbugs

- Medication Reconciliation in Home Care

- Central Line Associated Blood Stream Infections

- Safe Surgery Saves Lives Surgical Safety Checklist

- Medication Reconciliation at Discharge

- Improving Patient Safety for the Critically III

\section{Virtual Learning Collaboratives:}

- Acute Myocardial Infarction

- Reduce Falls and Injury from Falls

For details visit www.saferhealthcarenow.ca 